\title{
De l'art de faillir pour devenir un homme : du Mythe de Sisyphe à La Pierre qui pousse
}

\section{Marielle Chauvin}

\section{(Q) OpenEdition \\ Journals}

Édition électronique

URL : http://journals.openedition.org/lcc/155

DOI : $10.4000 /$ lcc. 155

ISSN : 2430-4247

\section{Éditeur}

Université Aix-Marseille (AMU)

\section{Référence électronique}

Marielle Chauvin, « De l'art de faillir pour devenir un homme : du Mythe de Sisyphe à La Pierre qui pousse ", Les chantiers de la création [En ligne], 2 | 2009, mis en ligne le 15 janvier 2015, consulté le 08 avril 2020. URL : http://journals.openedition.org/lcc/155 ; DOI : https://doi.org/10.4000/lcc.155 


\section{De l'art de faillir pour devenir un homme : du Mythe de Sisyphe à « La pierre qui pousse » \\ Marielle CHAUVIN, Université Paris 8 Vincennes - Saint-Denis}

L’histoire de Sisyphe est celle d'une condamnation pour une faute commise : le héros a cru pouvoir duper la mort. Sa punition consiste à rouler un rocher dans le Tartare jusqu'au sommet d'une colline d'où la pierre redescendra immanquablement avant d'avoir atteint son but. Ce cycle infécond d'une action centrée autour d'une pierre (inféconde par nature) se fait pourtant philosophiquement fécond car porteur du concept même d'absurde. On peut suivre la trace de cette pierre, dans une nouvelle de l'Exil et le Royaume, recueil de nouvelles, paru en 1957, que clôt « La pierre qui pousse ». Cette nouvelle écrite plus de dix ans après le Mythe de Sisyphe (paru en 1942) se présente sous bien des aspects comme le miroir inversé de cet essai. En effet, l’absurdité de la situation imaginée alors par Camus est humaine avant d'être philosophique : ici les dieux sont craints et non défiés, la faillite est celle d'un corps humain dont la pierre, trop lourde, vient à bout et la fertilité même n’est pas conceptuelle, mais incarnée dans des rapports humains rendus possibles par cette faillite même.

Il ne s'agit pas de faire de l'essai philosophique et de la nouvelle, deux entités ontologiquement distinctes, mais de voir comment la transposition du châtiment sisyphéen dans un contexte humain livre une vision de la condition humaine libérée peut-être de son absurdité conceptuelle. Pour ce, je vous propose d'étudier conjointement les deux œuvres en tentant d'y détecter ce qui, dans l'œuvre de Camus, se dévoile peu à peu comme un art de la chute.

\section{De l'erreur tragique à la tragique erreur : mythes, héros et expiations comme origines de l'art de faillir}

\subsection{Aspects mythiques}

Le mythe de Sisyphe se présente comme une relecture philosophique par Camus d'une histoire appartenant à la mythologie grecque dans une réflexion sur l’Absurde. Le mythe tel qu'il est relaté par Camus rend compte d'un certain nombre de fautes qui sont autant d'erreurs de conduite commises par le héros à l'égard des dieux : « On lui reproche d’abord quelques légèretés avec les dieux. Il livra leurs secrets. [...] Il en fut puni dans les 
enfers » (Camus, 1942, 163). C'est bien une faute ou même des fautes qui ouvrent le chemin du mythe et de son expiation. Ce n'est pas pour autant que cette erreur est une faillite, une faiblesse : elle est volontaire. Camus utilise et détourne la faute sisyphéenne à des fins conceptuelles : « Son mépris des dieux, sa haine de la mort, sa passion pour la vie, lui ont valu ce supplice indicible où tout l'être s'emploie à ne rien achever » (Camus, 1942, 164). C’est parce que l'erreur est volontaire que ce mythe peut être support à une réflexion théorique sur l'Absurde comme concept philosophique existentiel. Inversement, l'absurde me semble être logé dans «La pierre qui pousse » au cœur même du mythe d'une façon presque grotesque. On croirait l'absurde tourné en dérision. En effet, D’Arrast, l'ingénieur français, arrive à Iguape au moment de la procession du Bon Jésus. Cette fête est l’occasion d'un rituel particulier relaté en ces termes :

Un jour, la bonne statue de Jésus, elle est arrivée de la mer, en remontant le fleuve. Des pêcheurs l'a trouvée. Que belle ! Que belle! Alors, ils l'a lavée ici dans la grotte. Chaque année c'est la fête. Avec le marteau, tu casses, tu casses des morceaux pour le bonheur béni. Et puis quoi, elle pousse toujours, toujours tu casses. C’est le miracle. (Camus, 1957, 169)

L’idée même d'une pierre fertile, qui pourrait pousser lorsqu'on la casse est absurde, mais cet absurde-là n'est pas l'Absurde, il nous renvoie plutôt à une forme de naïveté supérieure dont on peut trouver une forme d'exaltation dans la Naissance de la Tragédie de Nietzsche, ce dernier la percevant comme l'indice de l'instinct apollinien du Beau :

Pour supporter la vie, il fallait que les Grecs, par une nécessité profonde, créassent ces divinités. Et cette création, nous devons l'imaginer comme une lente émergence, comme l'œuvre de cet instinct apollinien du Beau qui est de l'ordre originel des Titans (...) Quand nous rencontrons le «naïf » en art, il convient d'y reconnaître l'effet suprême de la civilisation apollinienne qui, d'abord, doit toujours renverser des Titans et terrasser des monstres et, par des puissants mirages et de joyeuses illusions, se rendre maîtresse du péril de sa profondeur et de sa capacité à souffrir. (27-9)

Les Titans eux-mêmes ne s’armèrent-ils pas de gigantesques rochers arrachés aux montagnes pour lutter justement contre Zeus et les autres dieux de l'Olympe, ceux-là même dont Sisyphe se moque ?

\subsection{Des dieux}

Des dieux, Sisyphe n'en a pas peur, son erreur consiste justement à les défier en dévoilant leurs faiblesses allant jusqu'à se jouer du jour du jugement dernier. Le héros n'est pas dans 
un rapport de soumission craintive à l'ordre divin, mais dans un rapport d'égal à égal, car, quelle que soit sa punition, il est dans le secret des dieux. D’ailleurs, si Albert Camus corrobore la version selon laquelle Sisyphe était un simple mortel - «Si l'on en croit Homère, Sisyphe était le plus sage et le plus prudent des mortels. Selon une autre tradition cependant, il inclinait au métier de brigand »(Camus, 1942, 163) - dans la mythologie grecque, Sisyphe est aussi le fondateur de Corinthe, fils d'Enarété, mortelle, et d’Éole, luimême fils D'Hellen (dont le père est Zeus) et d'une nymphe. De ce fait, si l'on en croit cette mythique généalogie, son rapport aux dieux ne peut-être tout à fait celui d'un mortel. Dans « La pierre qui pousse », deux fêtes religieuses sont célébrées au cours du récit : la fête pour St Georges et la fête du Bon Jésus. Dans la description de la nuit de danse et de transe dédiée à St George, le martyr apparaît. Le coq dit à d'Arrast avant d'entrer en transe: " "Saint George arrive. Regarde, regarde”, souffla le coq dont les yeux s'exhorbitaient » (Camus, 1957, 169). Et plus loin, d'Arrast lui-même dans un état de semi-conscience, voit une Diane antique : «D’Arrast, fasciné par cette danse ralentie, contemplait la Diane noire lorsque le coq surgit devant lui, son visage lisse maintenant décomposé » (172).

Cet épisode relevant d'une ritualité ancestrale primitive qui précède le moment de la procession du Bon Jésus nous plonge dans un univers antique réactualisé. Nous ne pouvons pas ne pas penser aux Dionysies grecques durant lesquelles processions, concours de dithyrambes et transes se complétaient dans une ritualité profane qui est à l'origine de la représentation tragique. Il y a en effet dans les circonstances décrites par Camus, une montée de l'effet tragique qui laisse pressentir une inévitable chute. Chez Nietzsche, cette chute est celle des Titans, celle de l'esprit apollinien face à la démesure dionysiaque, chute qui vaut pour son combat fraternel qui constitue précisément l’art de la tragédie : « Ainsi la difficile liaison de l'apollinien et du dionysiaque dans la tragédie pourrait être symbolisée par l'alliance fraternelle des deux divinités » (Nietzsche 130).

\subsection{De l'effet tragique à la chute du tragique sur terre}

Sisyphe est puni pour son erreur de mortel ayant voulu dépasser sa condition. Pour ce, il est condamné à une peine absurde : rouler un rocher en haut d'une colline pour voir son effort toujours échoué. Le condamné sait que son effort est vain et s’y soumet en toute conscience. C’est cet état de conscience qui fait de ce mythe une tragédie. Camus écrit : «Si ce mythe est tragique, c’est que son héros est conscient» (Camus, 1942, 165). En 
effet, la tragédie ne s'accomplit vraiment que lorsque le héros prend conscience de cette destinée en train de s’accomplir. Aristote, dans la Poétique, nomme trois moments: péripétie, reconnaissance et événement pathétique. La reconnaissance la plus haute est selon le philosophe : «celle qui résulte des actes accomplis eux-mêmes » (Aristote, 1455 a, 110). Il s'agit donc bien d'une prise de conscience au sens où l'entend Camus. Dans Le mythe de Sisyphe, suite à cette qualification tragique du Mythe, Camus écrit : «L'ouvrier d'aujourd'hui travaille, tous les jours de sa vie, aux mêmes tâches et ce destin n'est pas moins absurde. Mais il n'est tragique qu'aux rares moments où il devient conscient » (Camus, 1957, 165). Si le travailleur pauvre conceptualisé par le philosophe en 1942 n’est donc conscient de son état que rarement, celui qui écrit « La pierre qui pousse » plus de dix ans après fait de ces ouvriers pauvres d'Iguape, des hommes plus que lucides sur l'exploitation de l'homme par l'homme.

Relevons cet échange entre l’ingénieur français et le coq :

(...) Mais tu es seigneur. Socrate me l'a dit.

Moi, non. Mais mon grand-père l'était. Son père aussi et tous ceux d'avant son père. Maintenant il n'y a plus de seigneur dans nos pays.

Ah ! dit le noir en riant, je comprends, tout le monde est seigneur.

Non, ce ne n'est pas cela. Il n'y a ni seigneurs ni peuple.

L'autre réfléchissait puis il se décida :

Personne ne travaille, personne ne souffre ?

Oui, des millions d'hommes.

Alors c'est le peuple. (161)

La prise de conscience rendue explicite par ce dialogue rend compte d'un fait : la tragédie n'est plus dans l'univers mythique des héros. Elle est descendue sur terre, parmi les hommes.

\section{La pierre, enjeu d'une fécondité intellectuelle}

\subsection{Le rocher de Sisyphe cathartique ou métaphysique?}

Revenons un instant sur ce moment de prise de conscience qui fait du Mythe de Sisyphe un instant tragique. Ce moment de lucidité est également le moment d'appropriation d'un destin, nommé «reconnaissance » par Aristote : «La reconnaissance - son nom même l'indique - est le retournement qui conduit de l'ignorance à la connaissance » (Aristote 101). L'enjeu de la reconnaissance au sens où l'entend Aristote dans La Poétique est ailleurs. L'enjeu d'une bonne tragédie se mesure surtout à son effet produit sur les 
spectateurs. Voilà comment Aristote le définit : «Et puisque le poète doit susciter le plaisir qui vient, à travers l'imitation, de la pitié et de la crainte il est manifeste qu'il doit composer de manière à faire naître ce plaisir des actes accomplis » (Aristote 105). Cet effet dit cathartique de l'œuvre est visible dans l'élaboration d'Albert Camus. En effet, Le mythe de Sisyphe est cathartique au sens où il permet à Camus de se libérer du sentiment de l'absurde en l'élevant au niveau conceptuel :

Mais Sisyphe enseigne la fidélité supérieure qui nie les dieux et soulève les rochers. Lui aussi juge que tout est bien. Cet univers désormais sans maître ne lui paraît ni stérile ni futile. Chacun des grains de cette pierre, chaque éclat minéral de cette montagne pleine de nuit, à lui seul forme un monde. (Camus, 1957, 168)

Le rocher de Sisyphe est la clé de voûte du système philosophique. Il est à l'origine de ce royaume minéral qui se laisse, et c'est peut-être là sa limite, contempler mais peu penser.

En effet, rien ne peut plus “pousser” dans cet univers conceptuel où ce qui était absurde a repris du sens. Ainsi, nous sommes bien dans un système métaphysique au sens littéral, c'est-à-dire au-delà de la nature, et la description de ce royaume étincelant mais désertique. Le châtiment prend d'ailleurs effet dans le Tartare, lieu minéral des enfers où sont enfermés les Titans mais si Sisyphe également. Dans l'acceptation de cette peine, Sisyphe échappe d'ailleurs à autre chose : l'erreur elle-même. En effet, si son effort est voué à l'échec, dans l'acceptation de cette expiation, il se délivre de l'erreur possible logée au cœur des activités humaines. Ici, surhomme absurde, son action se déroule désormais dans un cycle parfait, délivrée de l'errare humanum est.

\subsection{La pierre qui pousse, pierre philosophale?}

Si l'espace du Mythe de Sisyphe est un royaume minéral, c'est sur un littoral humide, luxuriant, tropical que nous amène Camus dans « La pierre qui pousse ». C'est d'ailleurs dans une grotte, haut lieu d'humidité que s’accomplit le miracle : celui d'une pierre qui pousse. L'idée d'une pierre fertile n'est pas si absurde qu'on a pu vouloir l'entendre en premier lieu. En effet, cette recherche est le centre d'intérêt de l'alchimie et représente le point d'aboutissement de ce qui était appelé le Grand Euvre. Cette pierre permettrait de transformer en or un métal vil en fusion dans lequel on la jetterait. La première étape de fabrication de cette pierre fertile était appelée «l'œuvre au noir » du nom de la couleur que prendrait la préparation, elle consiste en une dissolution du mercure et coagulation de souffre très difficile à obtenir. Ce lien nous amène véritablement au cœur de la matière, au 
cœur de la physis et donc à un renoncement de la contemplation métaphysique. Notons d’ailleurs que cette étape initiale est appelée également la mors philosophica comme si cette plongée au cœur de la matière ne pouvait qu'amener au renoncement idéaliste. Dans L'œuvre au noir ${ }^{1}$, Marguerite Yourcenar imagine le personnage de Zénon, un médecin et alchimiste du XVI siècle qui parcourt le monde et connaît une fin tragique. Dans une période d'immobilisme et de remise en question, Zénon revient à son principal sujet d'étude dont il s'est éloigné, le corps :

Quoi qu'il fît, sa méditation le ramenait au corps, son principal sujet d'étude. (...) Et pourtant, après tant d'années passées à anatomiser la machine humaine, il s'en voulait de ne pas s'être hasardé plus audacieusement dans l'exploration de ce royaume aux frontières de peau, dont nous nous croyons les princes, dont nous sommes prisonniers. (Yourcenar 218)

Si la pierre philosophale serait une substance capable de réaliser la transmutation des métaux vils en or, on pourrait également en extraire la Panacée capable de guérir tous les maux et d'accéder à l'immortalité. La poursuite philosophique d'une pierre fertile résonne donc bien avec la quête d'un corps sans maux, sans faillite, sans faiblesse. Le Zénon de L'œuvre au noir comprend l'absurdité de cette quête car ce corps dont l'alchimiste cherche à parfaire ce qu'il considère comme une erreur, sa finitude, ses faillites est justement le modèle même de la mécanique alchimiste. Pour comprendre ce paradoxe du corps dont la pierre philosophale est le point de tension, revenons un instant sur la phusis telle que l'entend Aristote. Chez le philosophe, la phusis est à la fois ce qui ne peut être séparé de ce qu'elle détermine elle-même comme être naturel, et qui trouve en elle la raison de son être tout en étant en même temps l'incarnation de la forme séparable par la pensée. En ce sens, la pierre philosophale est la métaphore paradoxale de la situation de l’homme toujours aspiré par l'essence, toujours ramené, et cela, par les mêmes chemins, à la finitude de son corps, lieu même de la parfaite alchimie et de la chute. Le Sisyphe noir de « La pierre qui pousse » va faillir justement par ce corps, lequel, à la différence du héros antique, ne lui permettra pas le détachement surhumain face à l’absurde.

\subsection{De la faillite du corps comme limite.}

Le corps qui fascine les alchimistes, les médecins, les scientifiques est un corps clinique dont les mouvements, les réactions et les symptômes se trouvent isolés des émotions et

\footnotetext{
${ }^{1}$ Marguerite Yourcenar, l’Oeuvre au Noir, Gallimard, Paris, 1968, 511 pages.
} 
intellectualisés pour l'exercice. Pourtant, le Zénon de l'Euvre au Noir voit peu à peu s’imposer à lui la puissance du non-vouloir dans ce qui donne vie aux corps :

Son pouls dont il avait si assidûment étudié les battements, ignorait tout des ordres émanent de sa faculté pensante, mais s'agitait sous la faculté des craintes et des douleurs auxquelles son intellect ne s'abaissait pas. (...) De même, une ou deux fois dans sa vie, avait jailli scandaleusement et malgré soi la source des larmes. Plus alchimistes qu'il ne l'avait jamais été lui-même, ses boyaux opéraient la transmutation des cadavres de bêtes ou de plantes en matière vivante, séparant sans son aide l'utile de l'inutile. (Yourcenar 220)

Pour revenir à présent à «La pierre qui pousse », c’est bien ce corps, celui du pénitent qui va faillir, détournant ce Sisyphe d’un destin héroïque. Le coq a réchappé du naufrage d'un petit pétrolier sur lequel il travaillait qui a pris feu suite à une erreur dont il n’est pas responsable, tient-il à le préciser à d'Arrast. Son canot de sauvetage renversé, il dérive et voit arriver ses dernières heures.

Apercevant au loin la lumière de l'église d'Iguape, il fait la promesse de porter sur sa tête une pierre de cinquante kilos lors de la procession de la fête du Bon Jésus. Le jour de la procession venue, l'ingénieur français suit pas à pas le chemin de croix du coq dont il voit petit à petit s’atténuer les forces jusqu’à un épuisement certain de ce corps puissant mais ayant atteint alors ses limites. Cette chute, cette faillite inévitable du corps fait l'objet d'une description minutieuse :

L'autre tremblait, la salive se remettait à couler de sa bouche tandis que, sur tout son corps, la sueur jaillissait littéralement. Il prit une respiration qu'il voulait profonde et s'arrêta court. Il s'ébranla encore, fit trois pas, vacilla. Et soudain, la pierre glissa sur son épaule, qu'elle entailla, puis en avant jusqu’à terre, tandis que le coq, déséquilibré, s’écroulait sur le côté. (Camus, 1957, 181-2)

Le coq est un homme dont le corps est la force de travail, le gagne-pain. Pourtant, celui-ci ne répond plus à cause d'une erreur qu'il a commise : à la fête de St Georges, la veille, il a dansé toute la nuit. Cette pulsion de danse, le coq l’a avoué à d’Arrast : «Viens à la danse. Et tu m'emmèneras après. Sinon, je resterai, je danserai, je ne pourrai peut-être pas m’empêcher » (161). Cette pierre est terrestre, lourde et pleine, elle nous montre que les limites de nos connaissances sont marquées par notre finitude. Se dessine alors un autre homme.

\section{Solitaire, exilé, solidaire : de l'art de faillir pour devenir un homme}

\subsection{Du rituel comme erreur}


Le coq cède à la tentation de cette nuit de danse. Il prend le risque de la fatigue et oublie pour un instant, la terrible épreuve qui l'attend le lendemain. Il choisit de participer au rituel collectif plutôt que de rentrer comme il l'avait dit au coq. Cette nuit en l'honneur de St George n'est ni plus ni moins que la description d'un rituel primitif de transe. Il me semble bon de convoquer pour un instant Wittgenstein et les Remarques sur le rameau d'or de Frazer. Le rameau d'or est un répertoire prodigieux de fêtes et de rituels primitifs écrit à la fin du $\mathrm{XIX}^{\mathrm{e}}$ siècle par un ethnologue écossais. Cette somme est à l'origine de l'anthropologie religieuse dans son déploiement contemporain tout en étant symptomatique de la position d'un Occidental du XIX ${ }^{\mathrm{e}}$ siècle sur une ritualité qu'il juge instantanément irrationnelle. Wittgenstein rend compte de la cécité de Frazer, au sens même des rituels.

L'ethnologue fait justement apparaître l'acte rituel comme une erreur :

Frazer dit qu'il est très difficile de découvrir l'erreur dans la magie - et c'est pour cela qu'elle se maintient si longtemps - parce que, par exemple, un sortilège destiné à faire venir la pluie se révèle certainement, tôt ou tard, efficace. Mais alors il est étonnant précisément que les hommes ne s'avisent pas plus tôt que, même sans cela, tôt ou tard, il pleut. (Wittgenstein, 1982, 14)

L'exemple utilisé par Wittgenstein montre que le sens même du rituel tel qu’il est pratiqué par le coq et ses pairs ne relève pas d'une croyance dans l'efficacité directe dudit rituel sur leur sort. Les gens des cases qui sont désignés dès le début du texte comme étant les plus pauvres parmi les pauvres savent que la célébration de St George ou du Bon Jésus ne changera pas, comme par magie ou miracle, leurs vies. De fait, le sens du rituel est à chercher ailleurs dans une manifestation de sens où c'est en fait l'acceptation consciente de cette faillite proprement humaine qui est érigée en art de vivre.

\subsection{L'art de faillir}

Si l'on cesse de considérer le rituel comme une erreur au sens où ces usagers et ces participants ne l'envisagent ni comme une pratique efficace ni comme une célébration symbolique, mais bien comme la participation à une forme de vie collective, la participation du coq aux danses en l'honneur de St Georges qui l'amènera ensuite à l'épuisement et au renoncement de sa promesse devient dès lors une erreur féconde.

En effet, vue sous cet angle, la promesse de porter une pierre de cinquante kilos lors de la procession relève d'un exploit qui le distinguerait et le séparerait de ses frères, de ses camarades. Au contraire, la participation aux danses est l'indice d'une appartenance à un groupe social donné, en l’occurrence celui des pauvres, celui des cases et en ce sens, il 
n’est pas étonnant que le coq dise à D’Arrast de se retirer car il n’est pas des leurs : « Sans bienveillance, comme s’il parlait à un étranger : «Il est tard, Capitaine, dit-il. Ils vont danser toute la nuit, mais ils ne veulent pas que tu restes maintenant » (Camus, 1957, 173). De plus, faire un exploit lors de la procession serait une prétention, le rapprocherait d'une nature divine comme le héros tragique en son temps épouse un destin surhumain. Le coq veut rester parmi les siens dans un état distinct du héros, séparation qui est aussi une soumission à un ordre qui nous dépasse. Si l'expiation du pénitent, de par la performance physique qu'elle demande, est une forme d'élévation de l'homme vers Dieu, le rituel de transe est un moment où le divin descend parmi les vivants. Rappelons-nous le coq qui s’écrit tout à coup : « Saint George arrive. Regarde, regarde » (169).

Il s’agit bien d’une pensée dans laquelle la chute, l'art de faillir, est fécond. Cependant, l'homme qui se soumet à ce moment-là n'est pas fier et triomphant comme le Sisyphe dévalant la colline derrière sa pierre, les hommes en transe sont figés, terrassés, ils poussent des cris et le coq épuisé est en larmes. Le moment du rituel est une expérience de pensée, de mors philosophica au sens alchimique du terme ou l'homme est conscient d'une séparation, d'une œuvre au noir entre lui et le monde, c'est un moment où il se voit voyant, conscient d'un écart avec le monde. Le moment de l'acte rituel est l'indice d'une adhésion à une forme de vie tout en étant l'expérience d'une altérité radicale dans son propre corps. Telle est la conclusion de Wittgenstein : «L'éveil de l'intellect en effet s'effectue par une séparation d'avec le sol originaire d'avec le fondement originel de la vie. (la naissance du choix), (La forme de l'esprit qui s’éveille est l'adoration) »(Wittgenstein, 1982, 25). L'erreur du coq, qui consiste à danser jusqu'à épuisement et qui l'amène au renoncement, devient philosophiquement féconde ouvrant la voie d'une autre recherche portant cette fois sur la condition humaine. Dans les Maitres fous de Jean Rouch, documentaire datant de 1956 portant sur des rituels de transe où l'homme noir, le soumis, l’oublié incarne l'homme blanc, l'autre, le colon dans des images de possession très violentes, intensément dérangeantes. A ce propos, Thiphaine Samoyault écrit dans « Au lieu de passage » :

Ce qui saisit d'effroi n'est pas le corps qui tremble, qui grimace ou qui bave mais bien la crainte très grande que l'on peut avoir « d'aller de l'autre côté ou au-delà ». Il $\mathrm{y}$ a eut une époque de la langue où «transi de vie, avant de renvoyer à l'état amoureux, a voulu dire mort. (Samouyault 57)

C’est entre la vie et la mort que se présente le coq, dans état transi qui va justement être dépassé par l'autre. 


\subsection{Du royaume des hommes}

Pour l'homme simple que représente le coq, la faillite du corps est vécue sur le terrain émotionnel dans un jeu entre adhésion et renonciation. Pour l'intellectuel, par contre, élevé à la quête du savoir, l'acceptation de l'erreur, d'une faillite est plus difficilement acceptable. L’ingénieur français énonce un état de solitude, un état d’incertitude et de doute qui l'amène à l'exil. La première fois, il répond ainsi au coq: "J’étais fier, maintenant je suis seul (...)» (Camus, 1957, 165). D’Arrast est l'intellectuel exilé qui n’a pas trouvé son royaume, qui n'a pas trouvé le bonheur et c’est là que l'on mesure tout l'écart, mais aussi tout le chemin parcouru entre Le mythe de Sisyphe et «La pierre qui pousse ».

En effet, Camus conclut son essai philosophique en ces termes : «La lutte ellemême vers les sommets suffit à remplir un cœur d'homme. Il faut s’imaginer Sisyphe heureux » (Camus, 1942, 168). Dans «La pierre qui pousse », il s'agit moins d'une ascension que de l'acceptation d'une chute nécessaire face à laquelle la soif du savoir semble bien aisée. L’ingénieur français est celui qui cherche sa place, qui cherche à comprendre comment devenir un homme et c'est très justement la chute du coq, son renoncement qui va lui donner l'occasion de le découvrir. En effet, au moment de la chute du coq où celui-ci prend conscience qu'il n'est pas en mesure de continuer la procession et de mener à bien sa pénitence, d'Arrast, jusqu'alors réservé, fait lui aussi un choix soudain : « D’Arrast le regardait, sans trouver ses mots. Il se tourna vers la foule, au loin, qui criait à nouveau. Soudain, il arracha la plaque de liège des mains qui la tenaient et marcha vers la pierre » (Camus, 1957, 183). Continuant la procession, l’ingénieur commet alors une erreur volontaire, il se détourne de l'église et va déposer sciemment la pierre dans le quartier du coq. D’Arrast prend acte de la fécondité de l'erreur du coq et la prolonge. Se détourner de l'église ce n'est pas tant injurier les dieux que d'accepter d'être un homme. Camus écrit alors : «Et là, redressant toute sa taille, énorme soudain, aspirant à pleine goulée désespérées, l’odeur de misère et de cendre qu’il reconnaissait, il écouta monter en lui le flot d'une joie obscure et haletante qu'il ne pouvait nommer. » (Camus, 1957, 185).

Avec ce geste, non seulement d'Arrast replace le monde humain parmi les hommes et passe de l'exil au royaume mais il entre également de plein pied dans le sens métaphorique de la pierre philosophe qui est celui de l'amour puisque tout ce qui est touché par l'amour devient de l'or. Il est possible alors de retrouver le seul vrai paradis, celui que l'on a perdu : l’autre. 
Au terme de cette aventure, le frère du coq s’adresse à l'ingénieur en ces termes : «Assieds-toi avec nous » (Camus, 1957, 185). Une place a été faite parmi les hommes à l'intellectuel qui, exigeant d'abord la connaissance comme un dû, accepte d'échouer, de commettre des erreurs humaines et d'en devenir fécond. Peut-être que, face à une recherche à laquelle nous sommes tous confrontés devrions-nous toujours nous poser la question non pas de notre capacité à l'atteindre, mais bien de notre résignation à s’y soumettre. Pour Wittgenstein d'ailleurs la recherche philosophique se commet trop souvent dans une ascension conceptuelle alors qu'elle devrait se soumettre à une clarification des usages dans un exercice grammatical qui consiste à détecter les erreurs conceptuelles et à descendre au plus près (c’est-à-dire à aspirer à une littéralité) des usages. La présence du premier homme et de l'histoire de la faute, à la prise de conscience du corps, en passant par la chute, l'exil et finalement la procréation est implicite et le fait même que le dernier écrit inachevé de Camus soit précisément Le premier homme nous confirme dans cette voie. L'erreur d'Adam et Eve est féconde au sens le plus littéral qui soit. Dans la série justement appelée « Paradise Lost » du photographe français d’origine slovène, Klavdij Sluban, on trouve un inattendu Sisyphe. Le fait que cette série de photographies prises à Jérusalem et aux Caraïbes fut une commande pour une association française engagée pour la paix, montre bien que le paradis perdu est bien la pierre philosophale de l'amour. Wittgenstein dirait dans L'intérieur et L'extérieur : "Au lieu de "attitude envers l'âme », on pourrait aussi bien dire : « attitude envers l'homme » (Wittgenstein, 2000, 55). Un seul indice, m’a confié le photographe, Sisyphe est haïtien. Celui-ci est donc un frère du coq et ses pieds nus témoignent qu'il n'essaie ni de tenir une promesse ni de défier la mort, il tente juste de survivre. Dans son refus de m'en dire plus sur les conditions d'existence d'une telle image, le photographe m’a rappelé le rôle essentiel de l'artiste. Ce rôle est celui déjà défini par Aristote dans La Poétique : «De ce qui a été dit résulte clairement que le rôle du poète est de dire non pas ce qui a réellement eu lieu mais ce à quoi on peut s'attendre conformément à la vraisemblance ou à la nécessité » (Aristote 98). Ce champ est aussi parfois appelé le possible. Dieu lui-même laisse ce champ ouvert à Adam en lui permettant d'accéder à l'arbre. Ne nous l'interdisons pas, car il est le seul lieu où la liberté humaine peut s’exercer : la liberté de se tromper, de tomber et de recommencer. 


\section{Ouvrages cités}

Aristote. La Poétique. Traduction française de Michel Magnien. Paris : Le livre de poche, 1990.

Camus, Albert. Le mythe de Sisyphe, essai sur l'absurde. Paris : Gallimard, 1942.

—_. « La pierre qui pousse », L'exil et le Royaume. Paris : Gallimard, 1957.

Frazer, James Georges. Le rameau d'or. Traduction française de Pierre Sayn et Henry Peyre. Paris : Robert Laffont, 1981.

Nietzsche, Frederik. La naissance de la tragédie. Traduction française de Cornélius Heim. Paris : Denoël, 1964.

Samoyault, Tiphaine. "Au lieu de passage : quelques remarques sur la transe », Vacarme n²46, hiver 2009 : 56-58.

Wittgenstein, Ludwig. Les remarques sur le rameau d'or de Frazer. Traduction française de Jean Lacoste. Paris : L'âge d'homme, 1982.

_. L'intérieur et L'extérieur, derniers écrits sur la philosophe de la psychologie, II. Traduction française de Gérard Granel. Mauvezin : Trans-Europ-Repress, 2000.

Yourcenar, Marguerite. L'œuvre au Noir. Paris : Gallimard, 1968.

\section{Site internet}

Klavdij Sluban : $\leq$ http://www.sluban.com/> 


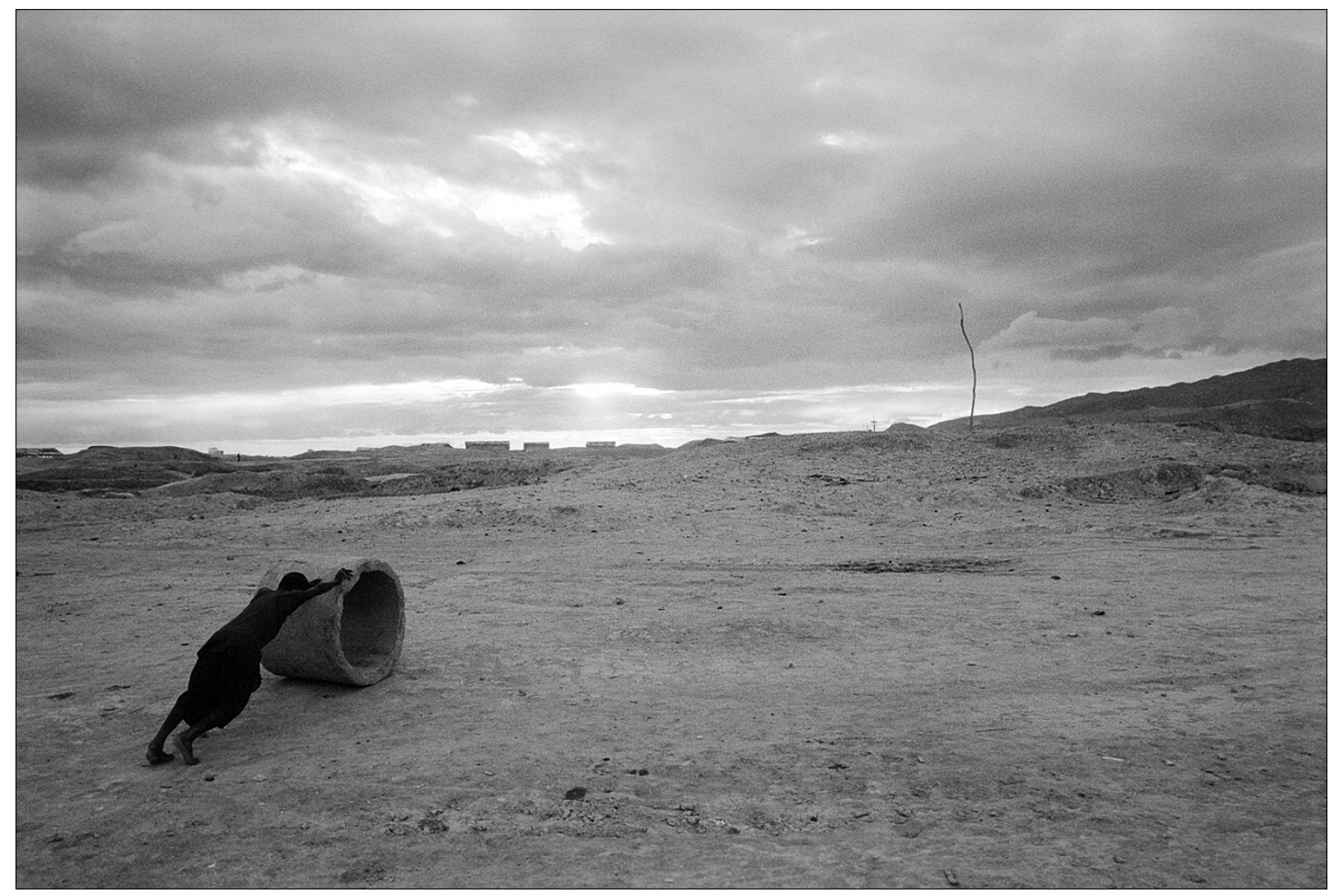

(CKlavdij Sluban, Paradise Lost, cycle en cours, 2000. (avec l'aimable autorisation de l'artiste) 